\title{
Femtosecond wave-packet dynamics in cesium dimers studied through controlled stimulated emission
}

\author{
Luqi Yuan, ${ }^{1}$ Gombojav O. Ariunbold ${ }^{1,2}$ Robert K. Murawski, ${ }^{3}$ Dmitry Pestov, ${ }^{4}$ Xi Wang, ${ }^{1}$ Anil K. Patnaik,,${ }^{1, *}$ \\ Vladimir A. Sautenkov, ${ }^{1,5}$ Alexei V. Sokolov, ${ }^{1}$ Yuri V. Rostovtsev, ${ }^{6}$ and Marlan O. Scully ${ }^{1,7,8}$ \\ ${ }^{1}$ Institute for Quantum Studies and Physics Department, Texas A\&M University, College Station, Texas 77843, USA \\ ${ }^{2}$ Department of Physics, National University of Mongolia, Ulaanbatar 210646, Mongolia \\ ${ }^{3}$ Department of Physics, Drew University, Madison, New Jersey 07940, USA \\ ${ }^{4}$ Department of Chemistry, Michigan State University, East Lansing, Michigan 48824, USA \\ ${ }^{5}$ P. N. Lebedev Institute of Physics, 53 Leninsky prospect, Moscow 119991, Russia \\ ${ }^{6}$ Department of Physics, University of North Texas, Denton, Texas 76203, USA \\ ${ }^{7}$ Applied Physics and Materials Science Group, Eng. Quad., Princeton University, Princeton, New Jersey 08544, USA \\ ${ }^{8}$ Max-Planck-Institute fur Quantenoptik, D-85748 Garching, Germany
}

(Received 3 March 2010; published 12 May 2010)

\begin{abstract}
We study the dynamics of wave packets in cesium dimers using a femtosecond-controlled pump-probe technique. We implement configurations with one pulse (pump) or two pulses (pump and control) to produce vibrational wave packets on the electronic excited state. The transmission of an additional, variable-delay probe pulse is measured to monitor the time evolution of the wave packets. In the case of the pump-control-probe configuration, a superposition of two independent wave packets is observed. In order to elucidate the observed experimental data, we develop a theory based on the Liouville equation for the density matrix associated with the Franck-Condon factors. Both the numerical and analytical calculations are in good agreement with our experimental results.
\end{abstract}

DOI: 10.1103/PhysRevA.81.053405

PACS number(s): 33.80.-b, 82.53.Hn, 42.50.-p

\section{INTRODUCTION}

Femtosecond dynamics of vibrational and rotational wave packets of dissociating molecular fragments in chemical reactions was first reported in Ref. [1]. Since then, the wave packet generation and evolution have been demonstrated and thoroughly studied for a number of diatomic molecules, including $\mathrm{I}_{2}[2,3], \mathrm{Na}_{2}[4,5], \mathrm{Li}_{2}[6,7]$, and many others [8,9]. Besides these successful research works, the two-color, pumpprobe laser experiment has been performed in Refs. [10-12]. The experiments with $\sim 100$-fs pulses have been used to investigate the dynamics of vibrational wave packets in $C^{1} \Pi_{u}$, $B^{1} \Pi_{u}$, and $D^{1} \Pi_{u}^{+}$electronic excited states in cesium dimers. In order to study the wave-packet dynamics in the $B^{1} \Pi_{u}$ state in Ref. [12], the wave packets have been produced by exciting the $\mathrm{Cs}_{2}$ dimer with a pump pulse from the $X^{1} \Sigma_{g}^{+}$ ground state to the target state and then photoionizing them into $\mathrm{Cs}_{2}^{+}(X)$ state with a time-delayed probe pulse. All those experiments have been carried out with a single pair of pump-probe pulses. It has been shown elsewhere that one can control the wave-packet dynamics using a sequence of pump, control, and probe fields [13-16]. In experiments with copropagating pump and control pulses, wave packets were shown to interfere coherently throughout an extended sample $[17,18]$.

Wave-packet dynamics has also been explored theoretically [19]. Density matrix formalism [20] has been adopted to describe the observables in the femtosecond pump-probe experiments [21-24]. Franck-Condon factors are related to the overlap integrals between vibrational wave functions and give

\footnotetext{
*Current Address: Air Force Research Laboratory, Wright-Patterson AFB, OH 45433.
}

rise the relative transition probabilities [25]. They have been used in Refs. [11,12,26] to explain their experimental results. However, a theory associated with the Liouville-von Neumann equation for the density matrix with the Franck-Condon factors has not yet been developed in detail for some generalized experimental conditions.

In this work, we present an all-optical alternative to the conventional photoionizing detection scheme [10-12] to monitor the femtosecond wave-packet dynamics. Namely the $\Lambda$-type configuration for energy levels is considered. That is, after the pump pulse creates the wave packet in the excited electronic state, the probe pulse stimulates this excitation down to some vibrational levels in the ground state. We use this method to visualize wave-packet time evolution in $B{ }^{1} \Pi_{u}$ state in $\mathrm{Cs}_{2}$, as is reported briefly in Ref. [27]. Furthermore, we extend it to pump-control-probe case by adding a control pulse. Instead of collinear setup as in Refs. [17,18], we use noncollinear beam geometry in both femtosecond pump-probe and pump-control-probe experiments. It does not require subfemtosecond stabilization of the time delay between pump and control pulses and allows for straightforward spatial filtering of the probe beam. To explain the observed results, we develop a detailed theoretical model based on the timedependent density matrix equations with the Franck-Condon factors. Both numerical simulations and analytical calculations for transmission of probe pulse are presented. These results are compared with experimental data.

The organization of the article is as follows. The pumpprobe and pump-control-probe experimental setup is discussed in Sec. II. In Sec. III, a theoretical model based on $\Lambda$-type system is presented and an analytical solution is obtained for the absorption of the femtosecond probe pulse assuming the pump and control excitations are independent of each other. The experimental and corresponding theoretical results on the 


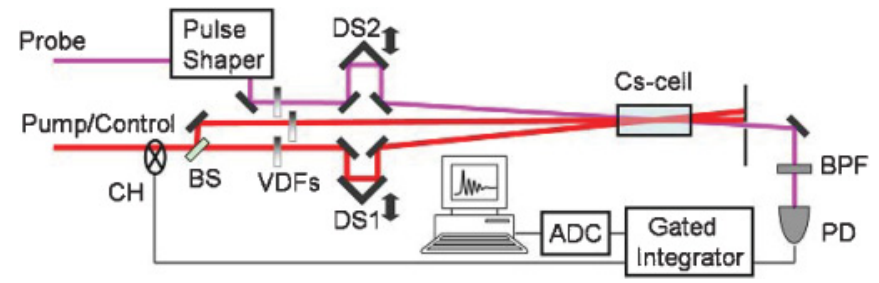

FIG. 1. (Color online) Experimental setup layout. $\mathrm{CH}$, chopper; BS, beam splitter; VDFs, variable neutral density filters; DS1,2 delay stages; BPF, variable bandpass filter such as a spectrometer with an adjustable exit slit; $\mathrm{PD}$, photodiode; ADC, analog-to-digital converter.

wave-packet dynamics are discussed in detail in Sec. IV. The results are summarized in $\mathrm{Sec}$. V.

\section{EXPERIMENTAL SETUP}

The experiments are performed using a commercially available femtosecond laser system (Legend, Coherent; $1 \mathrm{kHz}$ rep. rate) and an optical parametric amplifier (OPA; OperaVIS/UV, Coherent). The beam geometry at the sample and the signal acquisition arm are outlined schematically in Fig. 1. Briefly, the output of the regenerative amplifier is split into two uneven parts. The smaller fraction is used as a probe. The other part pumps the OPA, which produces pulses at $760 \mathrm{~nm}$ with the spectral full width at half maximum (FWHM) of $\sim 12 \mathrm{~nm}$. The $760-\mathrm{nm}$ beam is further split into two even fractions for pump and control delay lines. The probe pulse spectrum is centered at $805 \mathrm{~nm}$ and has FWHM of $31 \mathrm{~nm}$. To ensure delivery of transform-limited probe pulses at the sample, the probe beam passes through a pulse shaper, aided with the multiphoton intrapulse interference phase scan (MIIPS) algorithm that corrects for pulse phase distortions [28]. The pump and control pulse energies are $\sim 1 \mu \mathrm{J}(1 \mathrm{~mW}$ average power in each beam). The probe pulse energy is at least 10 -fold lower but is varied from one experiment to another to provide similar photon flux at the photodetector in the acquisition arm.

The three collimated but noncollinear beams are overlapped in a 3 -inch-long cesium cell, crossing under small angles $\left(\sim 1^{\circ}\right)$ to each other. Their $1 / e^{2}$ diameters, estimated by the knife edge method, are about $3 \mathrm{~mm}$ and $2.5 \mathrm{~mm}$ for pump (and control) and probe beams, respectively. Pump (and control) pulses induce $\mathrm{Cs}_{2}$ vibrations (wave packets) in the excited $B^{1} \Pi_{u}$ state, and probe pulses read out the wave-packet evolution by promoting excited molecules back into the ground $X^{1} \Sigma_{g}^{+}$ state. After the Cs cell, the probe beam is spatially and spectrally filtered and focused at a photodiode (DET-210, Thorlabs). For most of the experiments, only a small part of the full probe bandwidth reaches the detector. The signal from the photodiode is averaged by Gated Integrator (SR250, Stanford Research Systems). To retrieve the contribution due to the pump and/or control fields, we chop the pump or control beam at the half of the laser repetition rate (i.e., $\sim 500 \mathrm{~Hz}$ ) and subtract every other acquired shot. The resulting signal is digitized and recorded as a function of the probe pulse delay. It reflects the change in the transmitted energy of the probe pulse due to the motion of molecular wave packets.

The cell is heated up to $240^{\circ}-250^{\circ} \mathrm{C}$ with the temperature variation below $1^{\circ} \mathrm{C}$ for each particular measurement. These

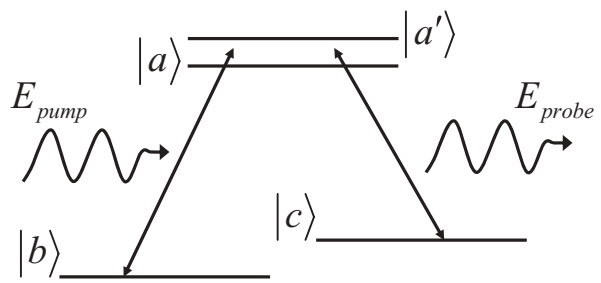

FIG. 2. Energy level diagram of a four-level system.

temperatures correspond to 1.2-1.9 mTorr pressure of Cs dimers or their concentration of $(2.3-3.6) \times 10^{13} \mathrm{~cm}^{-3}$ [29]. We estimate the interaction volume of the order of $0.1 \mathrm{~cm}^{3}$, i.e., $\sim 10^{12}$ of contributing $\mathrm{Cs}_{2}$ molecules.

\section{THEORETICAL MODEL}

First, we present the theoretical model of the pump-probe measurements. To calculate the absorption of the probe pulse in the pump-probe measurements, we choose a four-level system in which levels $|b\rangle$ and $|c\rangle$ are two vibrational levels in the ground state, $X^{1} \Sigma_{g}^{+}$, and levels $|a\rangle$ and $\left|a^{\prime}\right\rangle$ are two vibrational levels in the excited state, $B^{1} \Pi_{u}$ (Fig. 2). The Hamiltonian of this system is

$$
H=H_{0}+H_{I},
$$

where the unperturbed part of the Hamiltonian, $H_{0}$, and the interaction part of the Hamiltonian, $H_{I}$, are given by

$$
\begin{aligned}
H_{0}=\hbar \omega_{a}|a\rangle\left\langle a\left|+\hbar \omega_{a^{\prime}}\right| a^{\prime}\right\rangle\left\langle a^{\prime}\left|+\hbar \omega_{b}\right| b\right\rangle\left\langle b\left|+\hbar \omega_{c}\right| c\right\rangle\langle c| & \\
H_{I}= & -\left(\wp_{a b}|a\rangle\left\langle b\left|+\wp_{a c}\right| a\right\rangle\left\langle c\left|+\wp_{a^{\prime} b}\right| a^{\prime}\right\rangle\langle b|\right. \\
& \left.+\wp_{a^{\prime} c}\left|a^{\prime}\right\rangle\langle c|+\text { c.c. }\right) E(t)
\end{aligned}
$$

Here the vibrational level $v$ is displaced from the bottom of the potential well by $\hbar \omega$, which is defined as [30]

$$
\hbar \omega=\hbar \omega_{e}\left(v+\frac{1}{2}\right)-\hbar \omega_{e} \chi_{e}\left(v+\frac{1}{2}\right)^{2},
$$

where $\omega_{e}$ is the vibrational frequency and $\omega_{e} \chi_{e}$ is the vibrational anharmonicity. The electric dipole transition moment between vibrational level $v^{\prime}$ and vibrational level $v^{\prime \prime}, \wp^{\prime} v^{\prime \prime}$, is [31]

$$
\wp_{v^{\prime} v^{\prime \prime}}=\mu S\left(v^{\prime}, v^{\prime \prime}\right),
$$

where $\mu$ is the electronic transition moment. It is assumed to be constant, since the displacement of the nuclei from their equilibrium is relatively small (even at temperatures of $240^{\circ}-250^{\circ} \mathrm{C}$ ); the (smooth) $R$ dependence of the electronic matrix elements would provide higher-order corrections to our results. $S\left(v^{\prime}, v^{\prime \prime}\right)=\int \psi_{v^{\prime}}^{*}(R) \psi_{v^{\prime \prime}}(R) d \tau_{N}$ is the overlap integral between the two vibrational states $\psi_{v^{\prime}}$ and $\psi_{v^{\prime \prime}}$ in their respective electronic states. Here, $R$ is the internuclear distance and $d \tau_{N}$ is the element of volume of the space of the nuclear coordinates [32]. So $\left|S\left(v^{\prime}, v^{\prime \prime}\right)\right|^{2}$ is the Franck-Condon factor. The electronic transition between levels $|a\rangle$ and $|b\rangle$ and that between levels $\left|a^{\prime}\right\rangle$ and $|b\rangle$ are near resonant with the frequency of the pump pulse. Also the electronic transition between levels $|a\rangle$ and $|c\rangle$ and that between levels $\left|a^{\prime}\right\rangle$ and $|c\rangle$ are near resonant with the frequency of the probe pulse. After 
calculating the absorption of the probe pulse in this four-level system, vibrational levels in the ground and excited states are rechosen and the calculations are repeated for another set of vibrational levels. The total absorption of the probe pulse is defined as a sum over all possible absorption (emission) paths for such four-level configurations.

Because of the large relaxation time of cesium dimer ( $\sim 1 \mathrm{~ns}$ ), the equation of motion for the density matrix is [20]

$$
\dot{\rho}=-\frac{i}{\hbar}[H, \rho]
$$

Since the durations of the pump pulse and the probe pulse $(<100 \mathrm{fs})$ are much smaller than the time delay of the probe pulse ( $>1 \mathrm{ps}$ ), we can calculate the corresponding molecular polarizations induced by them separately. Let us consider one radiation field, $E_{i}(t)=E_{i}^{0} \cos \left(v_{i} t+\varphi_{i}\right)$, where $i=p$ for pump and $i=p r$ for probe, and do the rotation-wave approximation (RWA)

$$
\begin{aligned}
\rho_{a b} & =\tilde{\rho}_{a b} e^{-i v_{i} t-i \varphi_{i}}, \\
\rho_{a c} & =\tilde{\rho}_{a c} e^{-i v_{i} t-i \varphi_{i}}, \\
\rho_{a^{\prime} b} & =\tilde{\rho}_{a^{\prime} b} e^{-i v_{i} t-i \varphi_{i}}, \\
\rho_{a^{\prime} c} & =\tilde{\rho}_{a^{\prime} c} e^{-i v_{i} t-i \varphi_{i}} .
\end{aligned}
$$

We can write down the density matrix elements explicitly as

$$
\begin{aligned}
& \dot{\tilde{\rho}}_{a b}=-i\left(\omega_{a b}-v_{i}\right) \tilde{\rho}_{a b}-\frac{i}{\hbar} \frac{\wp_{a b} E_{i}^{0}}{2}\left(\rho_{a a}-\rho_{b b}\right) \\
& +\frac{i}{\hbar} \frac{\wp_{a c} E_{i}^{0}}{2} \rho_{c b}-\frac{i}{\hbar} \frac{\wp_{a^{\prime} b} E_{i}^{0}}{2} \rho_{a a^{\prime}}, \\
& \dot{\tilde{\rho}}_{a c}=-i\left(\omega_{a c}-v_{i}\right) \tilde{\rho}_{a c}-\frac{i}{\hbar} \frac{\wp_{a c} E_{i}^{0}}{2}\left(\rho_{a a}-\rho_{c c}\right) \\
& +\frac{i}{\hbar} \frac{\wp_{a b} E_{i}^{0}}{2} \rho_{b c}-\frac{i}{\hbar} \frac{\wp_{a^{\prime} c} E_{i}^{0}}{2} \rho_{a a^{\prime}}, \\
& \dot{\tilde{\rho}}_{a^{\prime} b}=-i\left(\omega_{a^{\prime} b}-v_{i}\right) \tilde{\rho}_{a^{\prime} b}-\frac{i}{\hbar} \frac{\wp_{a^{\prime} b} E_{i}^{0}}{2}\left(\rho_{a^{\prime} a^{\prime}}-\rho_{b b}\right) \\
& +\frac{i}{\hbar} \frac{\wp_{a^{\prime} c} E_{i}^{0}}{2} \rho_{c b}-\frac{i}{\hbar} \frac{\wp_{a b} E_{i}^{0}}{2} \rho_{a^{\prime} a}, \\
& \dot{\tilde{\rho}}_{a^{\prime} c}=-i\left(\omega_{a^{\prime} c}-v_{i}\right) \tilde{\rho}_{a^{\prime} c}-\frac{i}{\hbar} \frac{\wp_{a^{\prime} c} E_{i}^{0}}{2}\left(\rho_{a^{\prime} a^{\prime}}-\rho_{c c}\right) \\
& +\frac{i}{\hbar} \frac{\wp_{a^{\prime} b} E_{i}^{0}}{2} \rho_{b c}-\frac{i}{\hbar} \frac{\wp_{a c} E_{i}^{0}}{2} \rho_{a^{\prime} a}, \\
& \dot{\rho}_{b c}=i \omega_{c b} \rho_{b c}+\frac{i}{\hbar} \frac{\wp_{b a} E_{i}^{0}}{2} \tilde{\rho}_{a c}+\frac{i}{\hbar} \frac{\wp_{b a^{\prime}} E_{i}^{0}}{2} \tilde{\rho}_{a^{\prime} c} \\
& -\frac{i}{\hbar} \frac{\wp_{a c} E_{i}^{0}}{2} \tilde{\rho}_{b a}-\frac{i}{\hbar} \frac{\wp_{a^{\prime} c} E_{i}^{0}}{2} \tilde{\rho}_{b a^{\prime}}, \\
& \dot{\rho}_{a a^{\prime}}=i \omega_{a^{\prime} a} \rho_{a a^{\prime}}+\frac{i}{\hbar} \frac{\wp_{a b} E_{i}^{0}}{2} \tilde{\rho}_{b a^{\prime}}+\frac{i}{\hbar} \frac{\wp_{a c} E_{i}^{0}}{2} \tilde{\rho}_{c a^{\prime}} \\
& -\frac{i}{\hbar} \frac{\wp_{b a^{\prime}} E_{i}^{0}}{2} \tilde{\rho}_{a b}-\frac{i}{\hbar} \frac{\wp_{c a^{\prime}} E_{i}^{0}}{2} \tilde{\rho}_{a c}, \\
& \dot{\rho}_{a a}=\frac{i}{\hbar}\left(\frac{\wp_{a b} E_{i}^{0}}{2} \tilde{\rho}_{b a}+\frac{\wp_{a c} E_{i}^{0}}{2} \tilde{\rho}_{c a}-\frac{\wp_{b a} E_{i}^{0}}{2} \tilde{\rho}_{a b}\right. \\
& \left.-\frac{\wp_{c a} E_{i}^{0}}{2} \tilde{\rho}_{a c}\right) \text {, }
\end{aligned}
$$

$$
\begin{aligned}
\dot{\rho}_{b b}= & \frac{i}{\hbar}\left(\frac{\wp_{b a} E_{i}^{0}}{2} \tilde{\rho}_{a b}+\frac{\wp_{b a^{\prime}} E_{i}^{0}}{2} \tilde{\rho}_{a^{\prime} b}-\frac{\wp_{a b} E_{i}^{0}}{2} \tilde{\rho}_{b a}\right. \\
& \left.-\frac{\wp_{a^{\prime} b} E_{i}^{0}}{2} \tilde{\rho}_{b a^{\prime}}\right), \\
\dot{\rho}_{c c}= & \frac{i}{\hbar}\left(\frac{\wp_{c a} E_{i}^{0}}{2} \tilde{\rho}_{a c}+\frac{\wp_{c a^{\prime}} E_{i}^{0}}{2} \tilde{\rho}_{a^{\prime} c}-\frac{\wp_{a c} E_{i}^{0}}{2} \tilde{\rho}_{c a}\right. \\
& \left.-\frac{\wp_{a^{\prime} c} E_{i}^{0}}{2} \tilde{\rho}_{c a^{\prime}}\right), \\
\dot{\rho}_{a^{\prime} a^{\prime}}= & \frac{i}{\hbar}\left(\frac{\wp_{a^{\prime} b} E_{i}^{0}}{2} \tilde{\rho}_{b a^{\prime}}+\frac{\wp_{a^{\prime} c} E_{i}^{0}}{2} \tilde{\rho}_{c a^{\prime}}-\frac{\wp_{b a^{\prime}} E_{i}^{0}}{2} \tilde{\rho}_{a^{\prime} b}\right. \\
& \left.-\frac{\wp_{c a^{\prime}} E_{i}^{0}}{2} \tilde{\rho}_{a^{\prime} c}\right) .
\end{aligned}
$$

The general equation for the absorption of this field is [24]

$$
\begin{aligned}
Q & =\int \dot{\mathcal{P}} E d t \\
& =\int\left(\wp_{a b} \dot{\rho}_{b a}+\wp_{a c} \dot{\rho}_{c a}+\wp_{a^{\prime} b} \dot{\rho}_{b a^{\prime}}+\wp_{a^{\prime} c} \dot{\rho}_{c a^{\prime}}+\text { c.c. }\right) E_{i}(t) d t
\end{aligned}
$$

After RWA and using the approximation $\dot{\tilde{\rho}} \ll v_{p r} \tilde{\rho}$, this absorption equation will turn to

$$
\begin{aligned}
Q= & 2 \operatorname{Im} \int v_{p r}\left(\frac{\wp_{b a} E_{p r}^{0}}{2} \tilde{\rho}_{a b}+\frac{\wp_{c a} E_{p r}^{0}}{2} \tilde{\rho}_{a c}\right. \\
& \left.+\frac{\wp_{b a^{\prime}} E_{p r}^{0}}{2} \tilde{\rho}_{a^{\prime} b}+\frac{\wp_{c a^{\prime}} E_{p r}^{0}}{2} \tilde{\rho}_{a^{\prime} c}\right) d t .
\end{aligned}
$$

Therefore, the density matrix elements can be found when the pump pulse with frequency $v_{p}$ and phase $\varphi_{p}$ comes in first if we use the Eqs. (11) to (20). Then these results can be the initial conditions for the calculation when the probe pulse with frequency $v_{p r}$ and phase $\varphi_{p r}$ comes in with the time delay $\Delta t$. Validity of this assumption rests on the fact that the decay time scale of $\mathrm{Cs}_{2}$ is too long ( $\left.\sim 1 \mathrm{~ns}\right)$ compared to probe delay time $\Delta t$. Matrix elements can be found with the Eqs. (11) to (20). The absorption can also be found by the Eq. (22) with the time delay. We have to average over all possible phases because of the noncollinear beam geometry. Pulses do not copropagate in our experiments. Even if the phase of each pulse is stable, it is still different in different parts of the sample. Therefore, we should average our results of absorption with different phases in each pairs of pulses to find our final numerical result.

Now let us consider how to find the analytical solution. First, we consider one pump pulse coming at time $t=0$ with the Gaussian pulse shape, $E_{p}(t)=E_{p}^{0} \cos \left(v_{p} t+\varphi_{p}\right)$, where $E_{p}^{0}=\varepsilon_{p} e^{-\frac{1}{2}\left(\frac{t}{\Delta \tau_{p}}\right)^{2}}$. Since $\rho_{b b} \approx \rho_{b b}^{0} \gg \rho_{a a}, \rho_{c b}$, and $\rho_{a a^{\prime}}$ where $\rho_{b b}^{0}$ is the initial condition, Eq. (11) can be approximated with

$$
\dot{\tilde{\rho}}_{a b} \approx-i\left(\omega_{a b}-v_{p}\right) \tilde{\rho}_{a b}+\frac{i}{\hbar} \frac{\wp_{a b} E_{p}^{0}}{2} \rho_{b b}^{0} .
$$


This equation with the first-order approximation can be solved easily and the result gives

$$
\tilde{\rho}_{a b}(t)=i \sqrt{2 \pi} \Delta \tau_{p} \rho_{b b}^{0} \frac{\wp_{a b} \varepsilon_{p}}{2 \hbar} e^{-\frac{1}{2} \Delta \tau_{p}^{2}\left(\omega_{a b}-v_{p}\right)^{2}} e^{-i\left(\omega_{a b}-v_{p}\right) t} .
$$

Similarly, solutions for matrix elements $\tilde{\rho}_{a c}(t), \tilde{\rho}_{a^{\prime} b}(t)$, and $\tilde{\rho}_{a^{\prime} c}(t)$ can be found. If we plug these results into Eq. (15) and take into account that the transitions between vibrational levels are near-resonant to the carrier frequencies of the corresponding pulses, this differential equation in the secondorder approximation will give

$$
\begin{aligned}
\rho_{b c}(t) \propto & -\rho_{b b}^{0}\left[\frac{\wp_{a c} \varepsilon_{p}}{\hbar} \frac{\wp_{b a} \varepsilon_{p}}{\hbar} e^{-\frac{1}{2} \Delta \tau_{p}^{2}\left(\omega_{a b}-v_{p}\right)^{2}}+\frac{\wp_{a^{\prime} c} \varepsilon_{p}}{\hbar}\right. \\
& \left.\times \frac{\wp_{b a^{\prime}} \varepsilon_{p}}{\hbar} e^{-\frac{1}{2} \Delta \tau_{p}^{2}\left(\omega_{a^{\prime} b}-v_{p}\right)^{2}}\right] e^{-\frac{1}{2} \Delta \tau_{p}^{2} \omega_{c b}^{2}} e^{i \omega_{c b} t} .
\end{aligned}
$$

Similarly, the coherent element $\rho_{a a^{\prime}}$ is

$$
\begin{aligned}
\rho_{a a^{\prime}}(t) \propto & \rho_{b b}^{0}\left[\frac{\wp_{a b} \varepsilon_{p}}{\hbar} \frac{\wp_{b a^{\prime}} \varepsilon_{p}}{\hbar} e^{-\frac{1}{2} \Delta \tau_{p}^{2}\left(\omega_{a^{\prime} b}-v_{p}\right)^{2}}+\frac{\wp_{b a^{\prime}} \varepsilon_{p}}{\hbar}\right. \\
& \left.\times \frac{\wp_{a b} \varepsilon_{p}}{\hbar} e^{-\frac{1}{2} \Delta \tau_{p}^{2}\left(\omega_{a b}-v_{p}\right)^{2}}\right] e^{-\frac{1}{2} \Delta \tau_{p}^{2} \omega_{a^{\prime} a}^{2}} e^{i \omega_{a^{\prime} a} t} .
\end{aligned}
$$

Then let us consider that the probe pulse, $E_{p r}(t)=E_{p r}^{0} \cos \left(v_{p r} t+\varphi_{p r}\right)$, comes at $t=\Delta t$. Here, $E_{p r}^{0}=$ $\varepsilon_{p r} e^{-\frac{1}{2}\left(\frac{t-\Delta t}{\left.\Delta \tau_{p r}\right)^{2}}\right.}$. With the results for coherent density matrix elements $\rho_{b c}$ and $\rho_{a a^{\prime}}$ [Eqs. (25) and (26)], the differential equation for $\tilde{\rho}_{a b}[\mathrm{Eq} .(11)]$ can be solved as

$$
\begin{aligned}
\tilde{\rho}_{a b}(t)= & i \sqrt{2 \pi} \Delta \tau_{p r}\left[\frac{\wp_{a b} \varepsilon_{p r}}{2 \hbar} \rho_{b b}^{0}+\frac{\wp_{a c} \varepsilon_{p r}}{2 \hbar} \rho_{c b}(\Delta t)-\frac{\wp_{a^{\prime} b} \varepsilon_{p r}}{2 \hbar}\right. \\
& \left.\times \rho_{a a^{\prime}}(\Delta t)\right] e^{-\frac{1}{2} \Delta \tau_{p r}^{2}\left(\omega_{a b}-v_{p r}\right)^{2}} e^{-i\left(\omega_{a b}-v_{p r}\right)(t-\Delta t)} \cdot
\end{aligned}
$$

Similar results can also be found for $\tilde{\rho}_{a c}(t), \tilde{\rho}_{a^{\prime} b}(t)$, and $\tilde{\rho}_{a^{\prime} c}(t)$. Plug these results into Eq. (22), make the superposition, and neglect the constant terms. Therefore, the analytical expression for the absorption of probe pulse at delay $\Delta t$ can be written as

$$
\begin{aligned}
Q(\Delta t) \propto & \sum_{a, a^{\prime}, b, c}\left[\left(\wp_{c a} \wp_{a b} e^{-\Delta \tau_{p r}^{2}\left(\omega_{a c}-v_{p r}\right)^{2}}\right.\right. \\
& \left.+\wp_{c a^{\prime}} \wp_{a^{\prime} b} e^{-\Delta \tau_{p r}^{2}\left(\omega_{a^{\prime} c}-v_{p r}\right)^{2}}\right) \\
& \times\left(\wp_{a c} \wp_{b a} e^{-\frac{1}{2} \Delta \tau_{p}^{2}\left(\omega_{a b}-v_{p}\right)^{2}}+\wp_{a^{\prime} c} \wp_{b a^{\prime}} e^{-\frac{1}{2} \Delta \tau_{p}^{2}\left(\omega_{a^{\prime} b}-v_{p}\right)^{2}}\right) \\
& \times e^{-\frac{1}{2} \Delta \tau_{p}^{2} \omega_{c b}^{2}} \sin \left(\omega_{c b} \Delta t\right) \\
& -\left(\wp_{c a} \wp_{a^{\prime} c} e^{-\Delta \tau_{p r}^{2}\left(\omega_{a c}-v_{p r}\right)^{2}}+\wp_{c a^{\prime}} \wp_{a c} e^{-\Delta \tau_{p r}^{2}\left(\omega_{a^{\prime} c}-v_{p r}\right)^{2}}\right) \\
& \times\left(\wp_{b a^{\prime}} \wp_{a b} e^{-\frac{1}{2} \Delta \tau_{p}^{2}\left(\omega_{a b}-v_{p}\right)^{2}}+\wp_{a b} \wp_{b a^{\prime}} e^{-\frac{1}{2} \Delta \tau_{p}^{2}\left(\omega_{a^{\prime} b}-v_{p}\right)^{2}}\right) \\
& \left.\times e^{-\frac{1}{2} \Delta \tau_{p}^{2} \omega_{a^{\prime} a}^{2}} \sin \left(\omega_{a^{\prime} a} \Delta t\right)\right]
\end{aligned}
$$

For study of the pump-control-probe measurements, we assume that two wave packets generated by pump and control pulses are independent because the population is still mostly located in the ground levels and the coherent elements are relatively small when the control pulse comes in after the pump pulse. Therefore, we can calculate these two wave

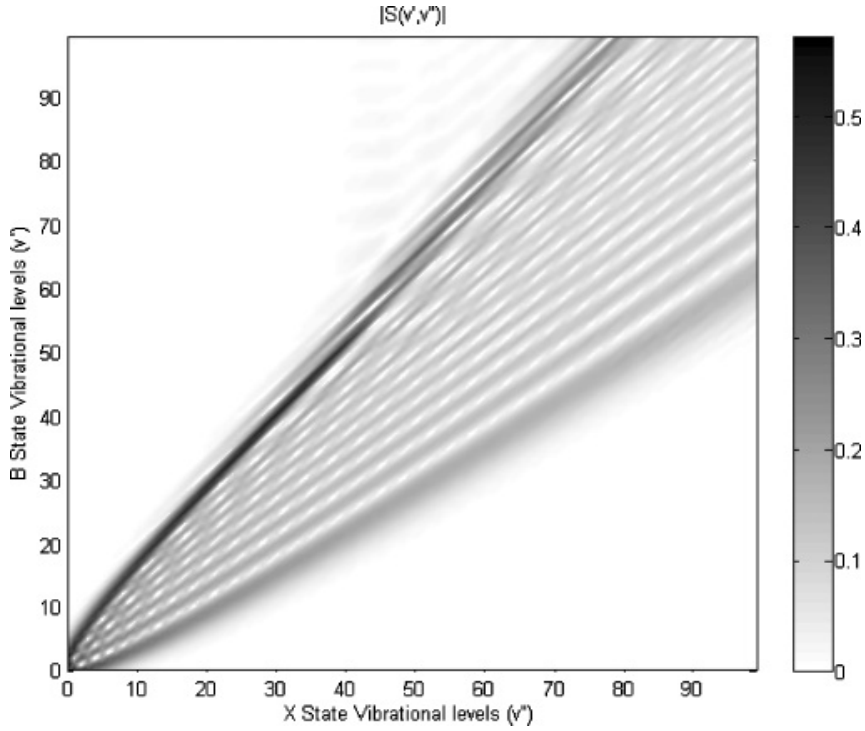

FIG. 3. $\left|S\left(v^{\prime}, v^{\prime \prime}\right)\right|$ where $v^{\prime \prime}$ is the vibrational level in the $X$ ground state and $v^{\prime}$ is the vibrational level in the $B$ excited state in cesium dimer.

packets separately by using the pump-probe model we show above.

\section{RESULTS AND DISCUSSION}

The Franck-Condon factors, $\left|S\left(v^{\prime}, v^{\prime \prime}\right)\right|^{2}$, can be found by using the exact eigenwave function for the Morse Potential [33]. Figure 3 shows the calculated absolute value of the overlap integral between one vibrational state in the ground state $X^{1} \Sigma_{g}^{+}$and that in the excited state $B^{1} \Pi_{u}$ in cesium dimer, $\left|S\left(v^{\prime}, v^{\prime \prime}\right)\right|$. The $\left(v^{\prime}, v^{\prime \prime}\right)$ transitions for which $v^{\prime} \sim$ $v^{\prime \prime}+3$ are coupled by the pump field and have the largest Franck-Condon factors. While the $\left(v^{\prime}, v^{\prime \prime}\right)$ transitions for which $v^{\prime \prime} \sim v^{\prime}+15$ are coupled by the probe field and still have the large Franck-Condon factors.

The parameters used in the theoretical calculations are chosen from those in the experiments. The initial conditions for the density matrix elements are all $\rho_{i j}^{0}=0$ except that $\rho_{b b}^{0}=\hbar \omega_{b} / k_{B} T$ and $\rho_{c c}^{0}=\hbar \omega_{c} / k_{B} T$. Here $k_{B} T \sim 356 \mathrm{~cm}^{-1}$.

Figure 4 shows the experimental result for the pumpprobe measurements. Probe pulse transmission is plotted as a function of its time delay relative to the pump pulse. The corresponding fast Fourier transform (FFT) spectra are given on the right panel. The same signal is recorded for spectral acquisition windows of variable width and exhibits different dephasing rates. In particular, the dephasing rate is maximal when the whole probe spectrum reaches the detector. It is significantly reduced, on the other hand, when the accepted FWHM bandwidth is narrow down to $1.5 \mathrm{~nm}$. Note that the probe pulse duration at the sample stays the same. All modifications are made in the acquisition arm, after the sample. The acquisition bandwidth, however, provides a straightforward means to adjust the number (subset) of contributing vibrational levels.

Numerical and analytical results for the small acquisition width limit are summarized in Fig. 5. They exhibit similar oscillations dominated by those at about $34 \mathrm{~cm}^{-1}$ and $68 \mathrm{~cm}^{-1}$, 

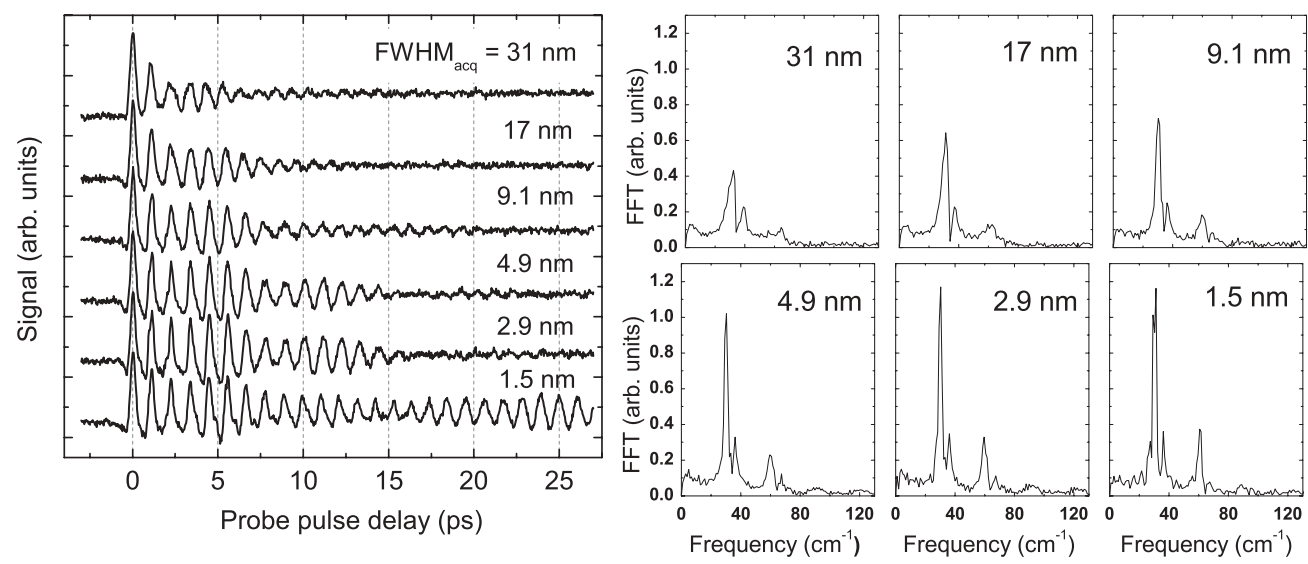

FIG. 4. The time dependence of the signal observed with one pump beam is shown on the left. The spectral width of the probe beam is varied from 31 to $1.5 \mathrm{~nm}$ by using a spectrometer with an adjustable exit slit. The observed temporal behavior of the spectrally selected wave packet is changed from a simple decay (probe $31 \mathrm{~nm}$ ) to decay and revival (probe $1.5 \mathrm{~nm}$ ). The FFT spectra of the recorded time-domain profiles are shown on the right.

i.e., at the vibrational frequency $\omega_{e}$ in the excited $B$ state and its overtone. This can be understood from the analytical result in (28). Because the near resonance of the pump pulse and the electronic transitions between vibration states $|a\rangle,\left|a^{\prime}\right\rangle$, and $|b\rangle$ and that of the probe pulse and the transitions between states $|a\rangle,\left|a^{\prime}\right\rangle$, and $|c\rangle$, the $\sin \left(\omega_{a^{\prime} a} \Delta t\right)$ term becomes much larger than the $\sin \left(\omega_{c b} \Delta t\right)$ term. Furthermore, due to the exponential functions in the equation, the term with frequency, $\omega_{a^{\prime} a}$, around the vibrational frequency of the $B$ excited state, $34 \mathrm{~cm}^{-1}$, contributes most to the signal while terms with higher-order harmonic frequency contribute less to the signal. Therefore, both experimental and theoretical results show a high peak at $34 \mathrm{~cm}^{-1}$ but a much lower peak at $68 \mathrm{~cm}^{-1}$. The dephasing of the oscillation amplitude is also shown in theory. It is the result of the vibrational anharmonicity term, $\omega_{e} \chi_{e}$. However, the dephasing rate from experiment and that from theory does not match quite well. The possible reason is that we do not include rotational levels in our model. Besides the anharmonicity of the molecular internuclear potential, the ro-vibrational coupling also does effects on the dephasing of the wave-packet motion [34].
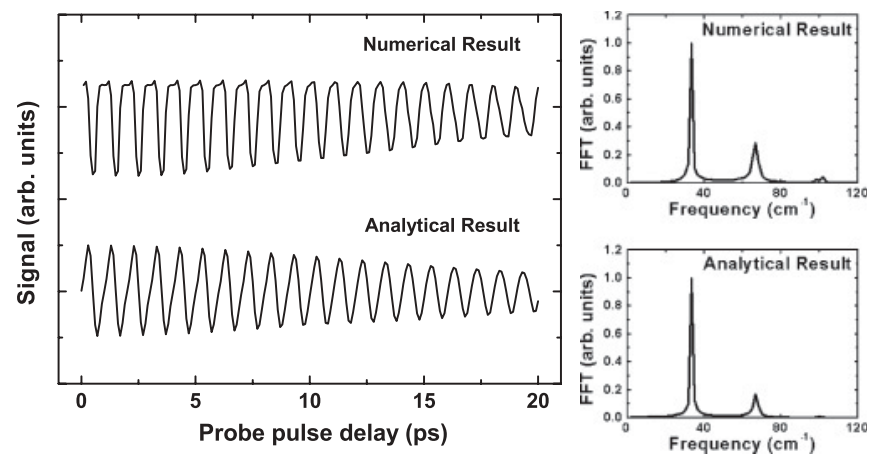

FIG. 5. Dependence of the absorptions of the probe pulse with width $31 \mathrm{~nm}$, calculated both with the numerical simulation and the analytical solution, on the probe pulse delay in pump-probe measurements. Corresponding FFT spectra are shown on the right.
Next, we evaluate the results of our pump-control-probe measurements and simulate the observed dynamics by extending the model above. We assume that two wave packets, generated by pump and control pulses, are independent. The experimentally recorded signal for different time delays between pump and control pulses is shown in Fig. 6. The time delay between pump pulse and control pulse is changing from 0 to $T$, where $T$ is the period of the signal oscillation in the pump-probe measurements, which is about $1 \mathrm{ps}$. The acquisition bandwidth used for observing the signal is $1.5 \mathrm{~nm}$. The superposition pattern between two wave packets in the cesium dimer can be seen. When the time delays between pump pulse and control pulse are 0 and $T$, two FFT peaks are found in the FFT spectra. One is at the frequency $34 \mathrm{~cm}^{-1}$ and the other one is at $68 \mathrm{~cm}^{-1}$, which are the same with the results in pump-probe measurements. However, when the time delays between pump pulse and control pulse are $T / 4$ and $3 T / 4$, only the peak at $34 \mathrm{~cm}^{-1}$ is surviving. When the delay time become half of the period, say $T / 2$, the first peak at $34 \mathrm{~cm}^{-1}$ disappears but the peak at the second-harmonic frequency $68 \mathrm{~cm}^{-1}$ appears again. Both numerical simulation (Fig. 7) and analytical calculation (Fig. 8) show the similar results with the experiment. Therefore, we are convinced that two wave packets generated by the pump and control pulses are independent and they produce the superposition pattern as shown above.

Such superposition pattern can also be read using the analytical result for pump-probe measurement [Eq. (28)]. Since two wave packets generated by pump and control pulses are independent, this superposition pattern shown above is the result of the superposition of the sine functions listed in this equation. When the time delays between pump pulse and control pulse are $T / 4$ and $3 T / 4$, the second-harmonic frequency terms, with frequency $68 \mathrm{~cm}^{-1}$ and period $T / 2$, are destructive. On the other hand, when this time delay becomes $T / 2$, the frequency terms, with frequency $34 \mathrm{~cm}^{-1}$ and period $T$, generate a destructive superposition. Therefore, with different time delays between pump and control pulses, FFT peaks appear or disappear accordingly. 

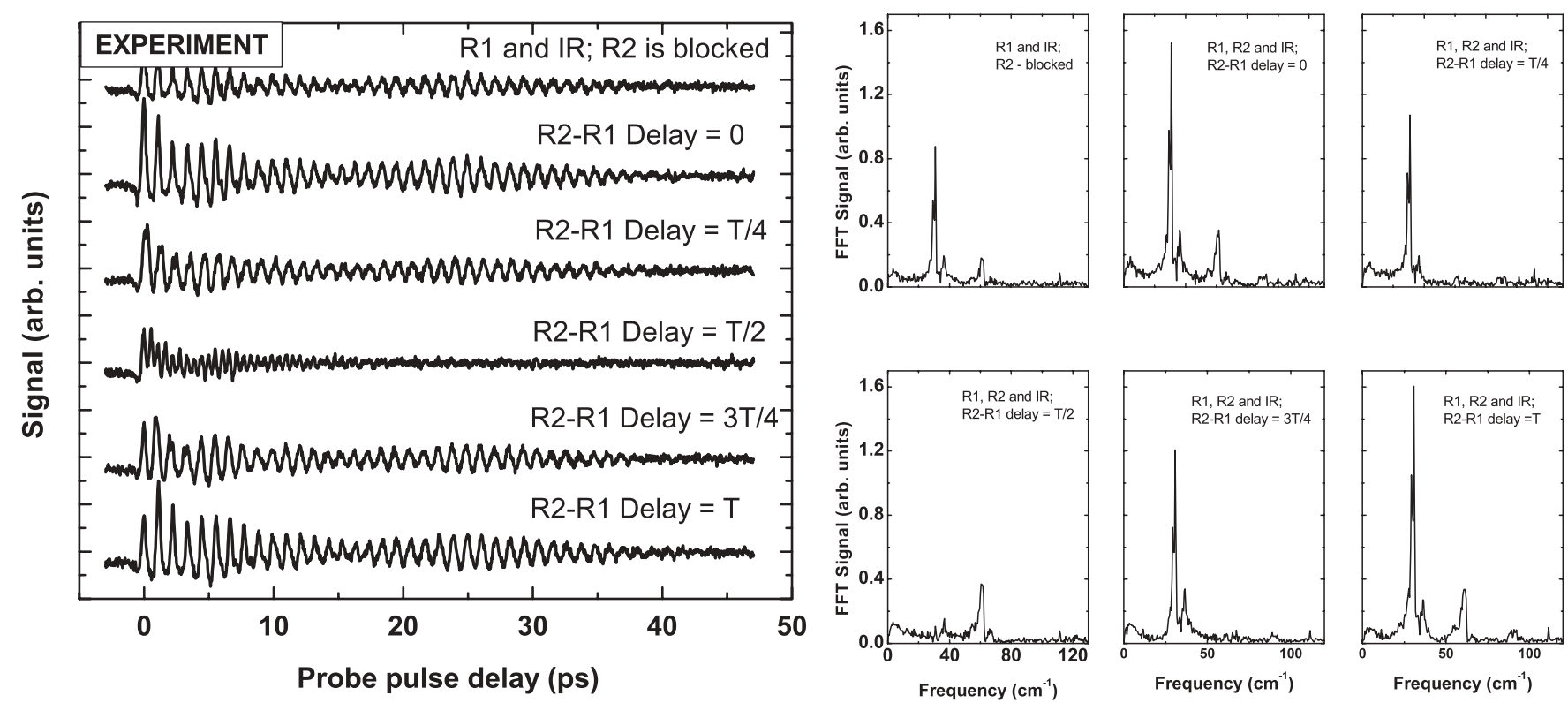

FIG. 6. Dependence of experimental signal on probe (IR) delay, with different time delay between pump (R1) and control (R2) in pump-control-probe measurements. FFT spectra are on the right.
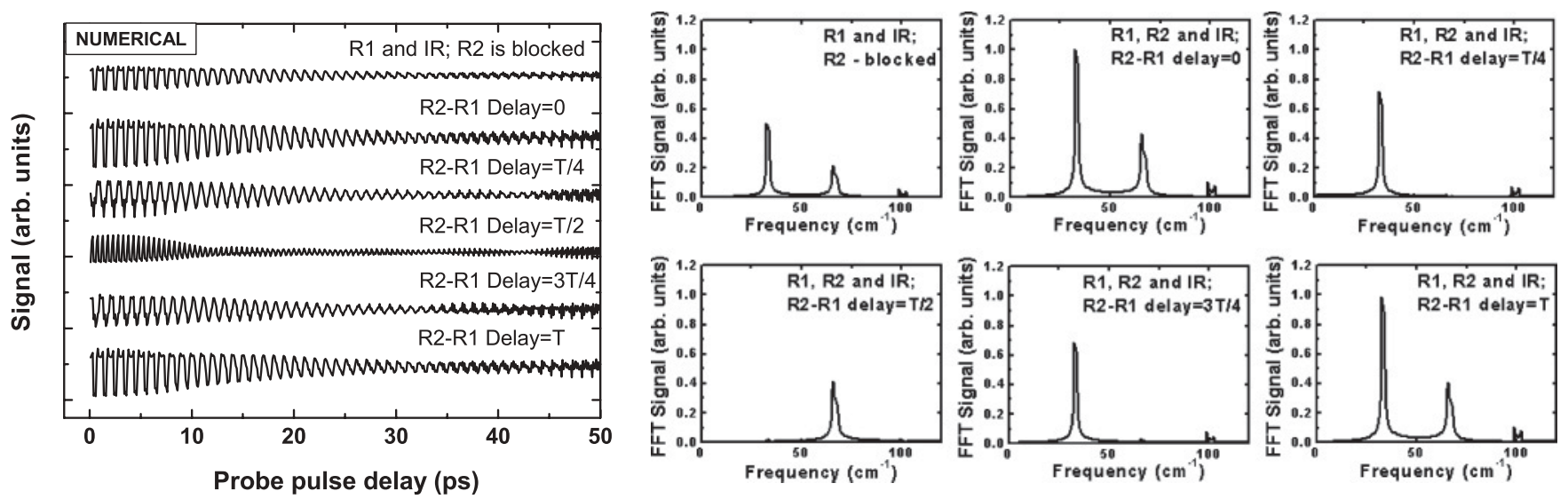

FIG. 7. Numerical results of pump-control-probe measurements with different time delay between pump pulse and control pulse. FFT spectra of the absorption signal are on the right.
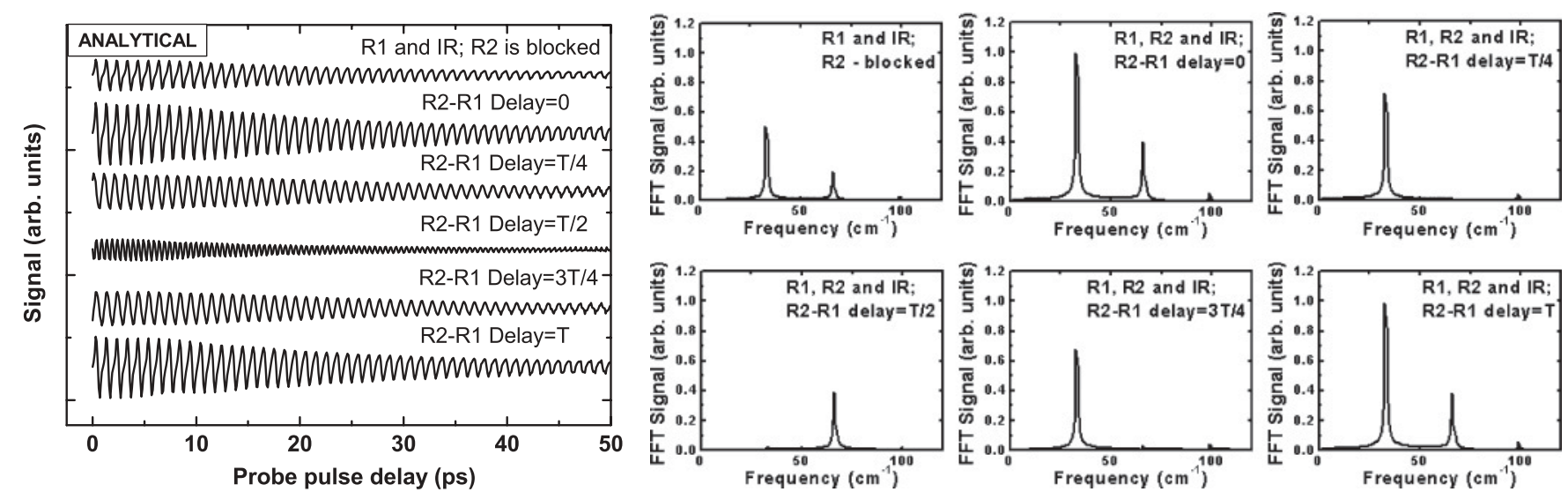

FIG. 8. Analytical results of pump-control-probe measurements with different time delay between pump pulse and control pulse. FFT spectra of the absorption signal are on the right. 
Interestingly, we can see the oscillation in the experiment shows some kind of "revival" at the large probe time delay (Fig. 6, on the left). Also, such "revival" is found in the numerical simulation results (Fig. 7), though at different delay time. However, it is not seen in the analytical solutions (Fig. 8). The reason may be that we neglect the weak wave packets in the ground state in theory for easy calculation and also we use a lot of approximations in finding the analytical solutions.

\section{CONCLUSION}

We study the wave-packet dynamics by using pumpprobe and pump-control-probe techniques associated with a $\Lambda$-type configuration. The transmission of the probe pulse is measured to monitor the wave-packet dynamics created either by one (pump) or two (pump and control) pulses into excited electronic state, $B{ }^{1} \Pi_{u}$, from the ground state, $X^{1} \Sigma_{g}^{+}$, in cesium dimers. The FFT spectra extracted from the time-dependent transmission traces for the probe pulse exhibit peaks at frequencies $34 \mathrm{~cm}^{-1}$ and $68 \mathrm{~cm}^{-1}$ that correspond to vibrational level splitting (and its overtone) in the excited state. It is also shown that a proper choice of time delay between pump and control pulses does alter the temporal characteristics of superposition of wave packets. In particular, at the delay equal to half-period of $34 \mathrm{~cm}^{-1}$ modulation, the main peak in the FFT spectrum is eliminated and the oscillations feature only the double-frequency component at $68 \mathrm{~cm}^{-1}$. For the pump-control delay matching the full time period of $34 \mathrm{~cm}^{-1}$ frequency splitting, however, the initial emission or absorption dynamics is restored.

The experimental observations are supported by a theoretical model based on density matrix formalism with the Franck-Condon factors. The theoretical results illustrate quite good agreement with the experimental data. In addition to numerical simulations, the simplified analytical treatment that elucidates the origin of probe pulse transmission modulation is also presented.

The presented theoretical method can be presumably applied to other similar experiments on ultrafast processes in diatomic molecules. This includes the study of coherent anti-Stokes Raman scattering in cesium dimers. We have plans for detailed simulation for such experiments.

\section{ACKNOWLEDGMENTS}

This work was supported by Office of Naval Research (Grants No. N00014-08-1-0948, and No. N00014-09-1-0888), the National Science Foundation (Grants No. PHY 354897 and No. 722800), the Texas Advanced Research Program (Grant No. 010366-0001-2007), the Army Research Office (Grant No. W911NF-07-1-0475), the CRDF, and the Robert A. Welch Foundation (Grants No. A1261 and No. A1547).
[1] A. H. Zewail, Science 242, 1645 (1988); M. Dantus, R. M. Bowman, and A. H. Zewail, Nature 343, 737 (1990).

[2] R. M. Bowman, M. Dantus, and A. H. Zewail, Chem. Phys. Lett. 161, 297 (1989).

[3] M. Gruebele, G. Roberts, M. Dantus, R. M. Bowman, and A. H. Zewail, Chem. Phys. Lett. 166, 459 (1990).

[4] T. Baumert, B. Bühler, R. Thalweiser, and G. Gerber, Phys. Rev. Lett. 64, 733 (1990).

[5] T. Baumert, M. Grosser, R. Thalweiser, and G. Gerber, Phys. Rev. Lett. 67, 3753 (1991).

[6] J. M. Papanikolas, R. M. Williams, P. D. Kleiber, J. L. Hart, C. Brink, S. D. Price, and S. R. Leone, J. Chem. Phys. 103, 7269 (1995).

[7] R. M. Williams, J. M. Papanikolas, J. Rathje, and S. R. Leone, J. Chem. Phys. 106, 8310 (1997).

[8] T. S. Rose, M. J. Rosker, and A. H. Zewail, J. Chem. Phys. 88, 6672 (1988).

[9] M. H. M. Janssen, R. M. Bowman, and A. H. Zewail, Chem. Phys. Lett. 172, 99 (1990).

[10] G. Rodriguez and J. G. Eden, Chem. Phys. Lett. 205, 371 (1993).

[11] G. Rodriguez, P. C. John, and J. G. Eden, J. Chem. Phys. 103, 10473 (1995).

[12] A. L. Oldenburg, P. C. John, and J. G. Eden, J. Chem. Phys. 113, 11009 (2000).

[13] J. J. Gerdy, M. Dantus, R. M. Bowman, and A. H. Zewail, Chem. Phys. Lett. 171, 1 (1990).

[14] E. D. Potter, J. L. Herek, S. Pedersen, Q. Liu, and A. H. Zewail, Nature 355, 66 (1992).
[15] V. Blanchet, M. A. Bouchene, O. Cabrol, and B. Girard, Chem. Phys. Lett. 233, 491 (1995).

[16] V. Blanchet, M. A. Bouchène, and B. Girard, J. Chem. Phys. 108, 4862 (1998).

[17] H. Katsuki, H. Chiba, B. Girard, C. Meier, and K. Ohmori, Science 311, 1589 (2006).

[18] A. Pe'er, E. A. Shapiro, M. C. Stowe, M. Shapiro, and J. Ye, Phys. Rev. Lett. 98, 113004 (2007).

[19] S. Mukamel, Principles of Nonlinear Optical Spectroscopy (Oxford University Press, New York, 1995).

[20] M. O. Scully and M. S. Zubairy, Quantum Optics (Cambridge University Press, Cambridge, England, 1997).

[21] S. Mukamel, Annu. Rev. Phys. Chem. 41, 647 (1990).

[22] S. H. Lin, R. Alden, R. Islampour, H. Ma, and A. A. Villaeys, Density Matrix Methods and Femtosecond Processes (World Scientific, Singapore, 1991).

[23] W. T. Pollard, H. L. Fragnito, J. Y. Bigot, C. V. Shank, and R. A. Mathies, Chem. Phys. Lett. 168, 239 (1990).

[24] R. K. Murawski, Y. V. Rostovtsev, Z.-E. Sariyanni, V. A. Sautenkov, S. Backus, D. Raymondson, H. C. Kapteyn, M. M. Murnane, and M. O. Scully, Phys. Rev. A 77, 023403 (2008).

[25] T. E. Sharp and H. M. Rosenstock, J. Chem. Phys. 41, 3453 (1964).

[26] M. Gruebele and A. H. Zewail, J. Chem. Phys. 98, 883 (1993).

[27] D. Pestov, A. Gombojav, X. Wang, R. K. Murawski, V. A. Sautenkov, Y. V. Rostovtsev, A. Patnaik, A. V. Sokolov, and M. O. Scully, in Conference on Lasers and Electro-Optics/ Quantum Electronics and Laser Science Conference and 
Photonic Applications Systems Technologies, OSA Technical Digest (CD) (Optical Society of America, Baltimore, MA, 2007), paper JThD34.

[28] B. Xu, J. M. Gunn, J. M. Dela Cruz, V. V. Lozovoy, and M. Dantus, J. Opt. Soc. Am. B 23, 750 (2006).

[29] A. N. Nesmeyanov, Vapor Pressure of the Chemical Elements, edited by G. Robert (Elsevier, Amsterdam/London, 1963).

[30] D. Steele and E. R. Lippincott, Rev. Mod. Phys. 34, 239 (1962).
[31] P. Atkins and R. Friedman, Molecular Quantum Mechanics, 4th ed. (Oxford University Press, New York, 2005).

[32] G. Herzberg, Molecular Spectra and Molecular Structure. I. Spectra of Diatomic Molecules, 2nd ed. (D. Van Nostrand Company, New York, 1950).

[33] P. M. Morse, Phys. Rev. 34, 57 (1929).

[34] S. Wallentowitz, I. A. Walmsley, L. J. Waxer, and Th. Richter, J. Phys. B 35, 1967 (2002). 